\title{
Deconfinement phase transition and the quark condensate
}

\author{
Christian S. Fischer ${ }^{1,2}$ \\ ${ }^{1}$ Institut für Kernphysik, Technische Universität Darmstadt, Schlossgartenstraße 9, \\ D-64289 Darmstadt, Germany \\ ${ }^{2}$ GSI Helmholtzzentrum für Schwerionenforschung GmbH, Planckstr. 1 D-64291 Darmstadt, Germany.
}

(Dated: October 31, 2018)

\begin{abstract}
We study the dual quark condensate as a signal for the confinement-deconfinement phase transition of QCD. This order parameter for center symmetry has been defined recently by Bilgici et al. within the framework of lattice QCD. In this work we determine the ordinary and the dual quark condensate with functional methods using a formulation of the Dyson-Schwinger equations for the quark propagator on a torus. The temperature dependence of these condensates serves to investigate the interplay between the chiral and deconfinement transitions of quenched QCD.
\end{abstract}

PACS numbers:

\section{Introduction}

The chiral and deconfinement transition of QCD is a subject of continuous interest. One of the unsolved problems is the question of an underlying mechanism relating both phenomena. Strictly speaking chiral and deconfinement phase transitions only occur in opposite sectors of the theory. The chiral condensate acts as order parameter for the chiral phase transition at vanishing quark masses, $m=0$, whereas the Polyakov loop signals center symmetry breaking at the deconfinement transition for $m \rightarrow \infty$. At intermediate masses both transitions develop a fascinating interplay [1], which is not yet understood in detail. Nonperturbative methods such as lattice gauge theory or functional methods are needed to explore this issue.

A recent development that sheds light on this connection is the investigation of spectral sums of the Dirac propagator and their behaviour under center transformations. Initiated by a work of Gattringer [2] spectral sums have been explored in Refs. [3, 4, 5, 6]. In particular it has been shown that the quark confinement mechanism is entirely encoded in the low lying spectral modes of the Dirac operator [6], which are also responsible for chiral symmetry breaking via the celebrated Casher-Banks relation. The spectral sums constitute order parameters for the deconfinement transition of QCD [2, 6].

In general, order parameters for deconfinement are not easily accessible by functional methods. In 7] a method has been developed to determine the Polyakov loop potential from the effective action, whereas in [8] the analytic structure of the quark propagator has been used to distinguish between the confined and deconfined phase. In this letter we report on a calculation of another order parameter for deconfinement, the dual quark condensate or 'dressed Polyakov loop' [5], with functional methods.

This dual condensate $\Sigma_{1}$ is defined by the Fouriertransform

$$
\Sigma_{1}=-\int_{0}^{2 \pi} \frac{d \varphi}{2 \pi} e^{-i \varphi}\langle\bar{\psi} \psi\rangle_{\varphi}
$$

of the ordinary quark condensate $\langle\bar{\psi} \psi\rangle_{\varphi}$ evaluated using $U(1)$-valued boundary conditions with angle $\varphi$ in the temporal direction of Euclidean, quenched QCD. Note that the usual antiperiodic boundary conditions for fermions require $\varphi=\pi$, whereas $\varphi=0$ corresponds to periodic boundary conditions; here we vary $\varphi$ in the interval $[0,2 \pi]$. In order to explain why the quantity $\Sigma_{1}$ is of considerable interest we note that the $\varphi$-dependent quark condensate $\langle\bar{\psi} \psi\rangle_{\varphi}$ can be represented by a sum over all possible closed chains of link variables, i.e. closed loops $l$, on a lattice. One obtains:

$$
\langle\bar{\psi} \psi\rangle_{\varphi}=\sum_{l} \frac{e^{i \varphi n(l)}}{m^{|l|}} U(l),
$$

where $U(l)$ denotes the closed chains of links including some sign and normalisation factors, see [5] for details. Each loop consists of $|l|$ links and is weighted with the corresponding power of the inverse quark mass $m$. Each time such a closed loop winds around the temporal direction of the lattice it picks up a factor $e^{ \pm i \varphi}$ from the $U(1)$-valued boundary condition introduced above. This leads to a weighting $e^{i \varphi n(l)}$, where $n(l)$ is the winding number of a given loop $l$. The dressed Polyakov loop $\Sigma_{1}$, Eq. (11), projects out loops with $n(l)=1$. It transforms under center transformation in the same way as the conventional Polyakov loop [9] and is therefore an order parameter for the deconfinement transition. The numerical agreement between dressed and conventional Polyakov loop has been established for gauge groups $S U(3)$ [10] and, remarkably, also for the centerless $G(2)$ 11].

In this work we determine the dual quark condensate from the Dyson-Schwinger equations (DSEs) of Landau gauge QCD at finite temperature 12]. However, we wish to point out that our method is sufficiently general to be of equal use in other functional approaches as e.g. the functional renormalisation group [13]. We evaluate the ordinary and the dual quark condensate from the trace of the quark propagator in a formulation of the DSEs on a torus. Our investigation of chiral symmetry breaking and deconfinement complements corresponding ones at vanishing temperature, see e.g. [14], and provides an interesting tool for further investigations of the QCD phase diagram by functional methods. 


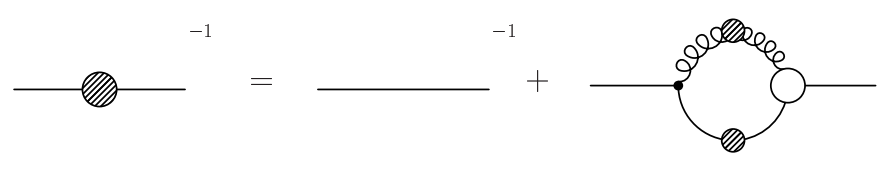

FIG. 1: The Dyson-Schwinger equation for the quark propagator. Filled circles denote dressed propagators whereas the empty circle stands for the dressed quark-gluon vertex.

\section{Dyson-Schwinger equations on a torus}

We work in Euclidean space with compact time and space directions, i.e. box size $V=L^{3} \times 1 / T$ with temperature $T$ and $1 / T<<L$. We choose periodic boundary conditions in the three spatial directions for the quark and gluon fields. The gluon field also obeys periodic boundary conditions in the temporal direction. For the fermion field $\psi$ we use the generalised, $U(1)$-valued boundary condition $\psi(\vec{x}, 1 / T)=e^{i \varphi} \psi(\vec{x}, 0)$ as described above. In loop integrals in momentum space this results in Matsubara modes $\omega_{p}\left(n_{t}\right)=(2 \pi T)\left(n_{t}+\varphi / 2 \pi\right)$ in the $p_{4}$-direction, which depend on the boundary angle $\varphi \in[0,2 \pi[$. In the spatial directions we have the usual Matsubara sums which are treated with the techniques described in [15].

In Euclidean momentum space at nonzero temperature the renormalised dressed quark propagator is given by

$$
S\left(\vec{p}, \omega_{p}\right)=\left[i \gamma_{4} \omega_{p} C\left(\vec{p}, \omega_{p}\right)+i \vec{p} A\left(\vec{p}, \omega_{p}\right)+B\left(\vec{p}, \omega_{p}\right)\right]^{-1}
$$

with vector and scalar quark dressing functions $C, A, B$. For the bare quark propagator $S_{0}$ we have $A=C=1$ and $B=Z_{m} m$, with bare quark mass $m$. The DSE for the quark propagator, shown in Fig. 1] reads

$$
S^{-1}\left(\vec{p}, \omega_{p}\right)=Z_{2} S_{0}^{-1}\left(\vec{p}, \omega_{p}\right)-C_{F} \frac{Z_{2}}{\widetilde{Z}_{3}} \frac{g^{2} T}{L^{3}} \sum_{n_{t}, n_{i}} \gamma_{\mu} S\left(\vec{k}, \omega_{k}\right) \Gamma_{\nu}\left(\vec{k}, \omega_{k}, \vec{p}, \omega_{p}\right) D_{\mu \nu}\left(\vec{p}-\vec{k}, \omega_{p}-\omega_{k}\right)
$$

where the sum is over temporal and spatial Matsubara modes. The Casimir $C_{F}=\left(N_{c}^{2}-1\right) / N_{c}$ stems from the colour trace; in this work we only consider the gauge group $S U(2)$. Furthermore, $D_{\mu \nu}$ denotes the gluon propagator in Landau gauge and $\Gamma_{\nu}$ the (reduced) quarkgluon vertex. The renormalisation factors $Z_{2}, Z_{m}$ and $\widetilde{Z}_{3}$ are determined in the renormalisation process.

In order to solve this equation we have to specify explicit expressions for the gluon propagator and the quarkgluon vertex. For the momentum range relevant for Eq. (4) we nowadays have very accurate solutions for the gluon propagator at zero temperature from both, lattice calculations and functional methods, see e.g. [16] and references therein. The temperature dependence of the gluon propagator, however, is much less explored. In [17] a combined lattice and DSE study records a different temperature dependence of the electric and magnetic sector. Whereas the magnetic part of the propagator seems to be indifferent to the deconfinement phase transition, the electric part is strongly increased at and around the critical temperature $T_{c} \approx 300 \mathrm{MeV}$. Although the lattice data still have considerable systematic errors they may correctly represent the qualitative temperature dependence of the gluon. We therefore use a temperature dependent fit to the data given by

\begin{tabular}{c||c|c|c|c}
$\mathrm{T}[\mathrm{MeV}]$ & 0 & 119 & 298 & 597 \\
\hline \hline$a_{T}(T)$ & 1 & 1 & 1.34 & 1.65 \\
$a_{L}(L)$ & 1 & 1 & 0.8 & 4.0
\end{tabular}

TABLE I: Temperature dependent fit parameter of Eq.(7).

$$
D_{\mu \nu}(q)=\frac{Z_{T}(q)}{q^{2}} P_{\mu \nu}^{T}(q)+\frac{Z_{L}(q)}{q^{2}} P_{\mu \nu}^{L}(q)
$$

with $q=\left(\vec{q}, \omega_{q}\right)$ and dressing functions $Z_{T}\left(\vec{q}, \omega_{q}\right)$ and $Z_{L}\left(\vec{q}, \omega_{q}\right)$. The transverse and longitudinal projectors with respect to the heat bath are given by

$$
\begin{aligned}
& P_{\mu \nu}^{T}(q)=\left(\delta_{i j}-\frac{q_{i} q_{j}}{\vec{q}^{2}}\right) \delta_{i \mu} \delta_{j \nu}, \\
& P_{\mu \nu}^{L}(q)=P_{\mu \nu}(q)-P_{\mu \nu}^{T}(q),
\end{aligned}
$$

with $(i, j=1 \ldots 3)$. The $S U(2)$ lattice results of Ref. [17] are well fitted by

$$
\begin{aligned}
Z_{T, L}\left(\vec{q}, \omega_{q}, T\right)= & \frac{q^{2} \Lambda^{2}}{\left(q^{2}+\Lambda^{2}\right)^{2}}\left\{\left(\frac{c}{q^{2}+\Lambda^{2} a_{T, L}(T)}\right)^{2}\right. \\
& \left.+\frac{q^{2}}{\Lambda^{2}}\left(\frac{\beta_{0} \alpha(\mu) \ln \left[q^{2} / \Lambda^{2}+1\right]}{4 \pi}\right)^{\gamma}\right\}
\end{aligned}
$$

with the temperature independent scale $\Lambda=1.4 \mathrm{GeV}$ and the coefficient $c=9.8 \mathrm{GeV}^{2}$. For gauge group $S U(2)$ we have $\beta_{0}=22 / 3$ and $\gamma=-13 / 22$ in the quenched theory and we renormalise at $\alpha(\mu)=0.3$. The temperature dependent scale modification parameters $a_{T, L}(T)$ are given in table I. In order to extend this fit to temperatures not given in the table we assume $a_{T, L}(T)$ to be temperature independent below $T=119 \mathrm{MeV}$ and only slowly rising above $T=597 \mathrm{MeV}$. For $T \in[119,597] \mathrm{MeV}$ we use cubic splines to interpolate smoothly between the values given in table I We expect the systematic error of this procedure to be of the same order as the systematic 
errors inherent in the lattice data. We also inherit the scale determined on the lattice using the string tension $\sqrt{\sigma}=0.44 \mathrm{GeV}[17]$.
For the quark-gluon vertex with gluon momentum $q=$ $\left(\vec{q}, \omega_{q}\right)$ and the quark momenta $p=\left(\vec{p}, \omega_{p}\right), k=\left(\vec{k}, \omega_{k}\right)$ we employ the following temperature dependent model

$$
\Gamma_{\nu}(q, k, p)=\widetilde{Z}_{3}\left(\delta_{4 \nu} \gamma_{4} \frac{C(k)+C(p)}{2}+\delta_{j \nu} \gamma_{j} \frac{A(k)+A(p)}{2}\right)\left(\frac{d_{1}}{d_{2}+q^{2}}+\frac{q^{2}}{\Lambda^{2}+q^{2}}\left(\frac{\beta_{0} \alpha(\mu) \ln \left[q^{2} / \Lambda^{2}+1\right]}{4 \pi}\right)^{2 \delta}\right) .
$$

where $\delta=-9 / 44$ is the anomalous dimension of the vertex. Note that because of $\gamma+2 \delta=-1$ the gluon dressing function together with the quark-gluon vertex behave like a running coupling at large momenta; this is a necessary boundary condition for any model interaction in the quark DSE. The dependence of the vertex on the quark dressing functions $A$ and $C$ is dictated by the SlavnovTaylor identity. The remaining fit function is purely phenomenological, see e.g. [18] where an elaborate version of such an ansatz has been used to describe meson observables. Here we use $d_{1}=7.6 \mathrm{GeV}^{2}$ and $d_{2}=0.5 \mathrm{GeV}^{2}$. A variation of these parameters shifts the critical temperatures of both, the chiral and the deconfinement transition but leaves all qualitative aspects of the results presented below unchanged. This is also true if one changes the deep infrared behaviour of the vertex to the (infrared divergent) form extracted in [14] and used in [18]; the confinement-deconfinement phase transition is insensitive to the question of scaling vs. decoupling in the sense specified in [16]. Details will be reported elsewhere.

The DSE is solved numerically using $n_{t}=8$ Matsubara frequencies and a three-volume of $V=(5 \mathrm{fm})^{3}$, which is large enough to avoid any significant volume effects. The renormalisation conditions are $C(\mu)=1$ and $B(\mu)=m$ with $\mu=(\vec{\mu}, \pi T)$ and $\vec{\mu}^{2}=20 \mathrm{GeV}^{2}$. The resulting quark dressing functions are subsequently used to determine the quark condensate according to

$$
\langle\bar{\psi} \psi\rangle_{\varphi}=\frac{4 Z_{2} N_{c} T}{L^{3}} \sum_{n_{t}, n_{i}} \frac{B\left(\vec{p}, \omega_{p}(\varphi)\right)}{\omega_{p}^{2}(\varphi) C^{2}+\vec{p}^{2} A^{2}+B^{2}},
$$

where we indicated the dependence of the frequencies on the generalised $U(1)$-boundary conditions. For nonvanishing bare quark masses $m$ this expression is divergent and, at least in the continuum limit, has to be renormalised accordingly. For the purpose of this letter, however, is is sufficient to work with the regularised expression at fixed ultraviolet cut-off corresponding to fixed lattice spacing.

\section{Numerical results}

First we explore the dependence of the quark condensate on the boundary angle $\varphi$ which can be read off Eq. (2). Each loop winding $n$ times around the temporal direction of the torus contributes a factor $\cos (n \varphi)$. Consequently the integrand in Eq. (11) can be expanded as a series in $\cos (n \varphi)$ and is symmetric in the interval $[0,2 \pi]$. This behaviour is clearly seen also in our numerical result for $\Delta_{\varphi} \equiv\langle\bar{\psi} \psi\rangle_{\varphi}$ shown in Fig 2 For $T=200 \mathrm{MeV}$ far below the deconfinement transition we find almost no angular dependence of the condensate. This is especially true for the heavier quark mass $m=60 \mathrm{MeV}$. For $T=400$ $\mathrm{MeV}$ far above the transition the behaviour is markedly different and we observe the characteristic cosine-type of behaviour of the condensate that we expect from Eq. (2). This is in nice agreement with the results of Ref. [5] on the lattice. The angular dependence of the condensate can be fitted well with a series in $\cos (n \varphi)$. Whereas for the heavier mass $m=60 \mathrm{MeV}$ terms with $n \leq 3$ are sufficient one needs at least terms up to $n \leq 7$ for the smaller mass $m=10 \mathrm{MeV}$. Thus the closer one gets to the chiral limit the more contributions from Polyakov loops with higher winding number around the temporal direction are significant. This is a direct consequence of the mass factor $1 / m^{|l|}$ in the expansion Eq. (2) and also seen on the lattice [19].

By far the largest contribution to the angular dependence of the condensate comes from loops with $n=1$ which are projected out by the Fourier transformation (11) to the dual condensate. The resulting temperature dependence of the dressed Polyakov loop is shown in Fig. 3 together with the conventional quark condensate and their derivatives. One clearly observes a change of behaviour in both, the conventional and the dual conden-

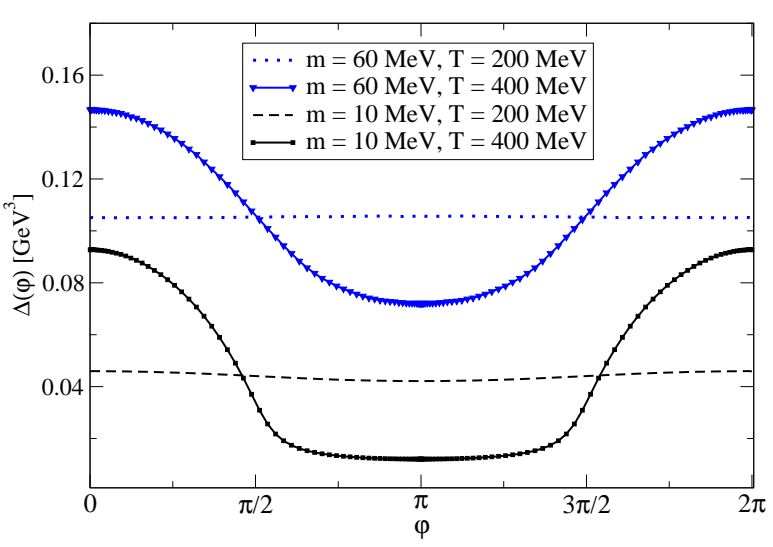

FIG. 2: The angular dependence of the quark condensate $\Delta_{\varphi} \equiv\langle\bar{\psi} \psi\rangle_{\varphi}$ below and above the deconfinement transition for two different quark masses. 


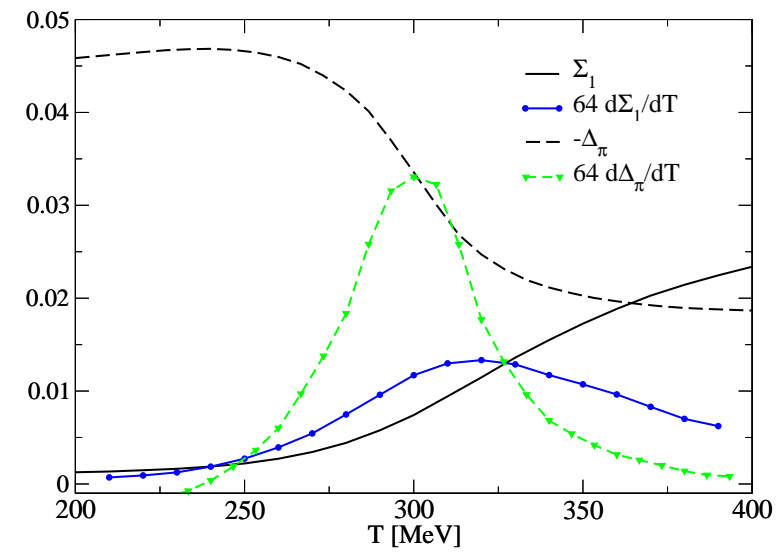

FIG. 3: The temperature dependence of the dressed Polyakovloop $\Sigma_{1}$ and the conventional quark condensate $\Delta_{\pi} \equiv$ $\langle\bar{\psi} \psi\rangle_{\varphi=\pi}$ together with their derivatives for $m=10 \mathrm{MeV}$.

sate above $T=270 \mathrm{MeV}$. The temperature derivative of both quantities has a peak in the region $T_{c}=300-320$ $\mathrm{MeV}$ signalling the chiral and deconfinement transition. Note that although both transitions are calculated from quantities with direct relation to the spectral properties of the quark propagator they do not necessarily give the same transition temperatures. On the contrary, the chiral transition occurs about $10-20 \mathrm{MeV}$ below the deconfinement transition. Whether the quantitative aspects of this difference is a model-independent result has to be investigated in more detail.

Finally we wish to point out that the absolute magnitude of our dressed Polyakov loop is about a factor of five smaller than the one calculated on the lattice [5]. Since the condensate and subsequently its dual is a renormalisation point dependent quantity we attribute this differ- ence to a yet undetermined renormalisation factor. This issue has to be clarified in future work. Furthermore we note that the dressed Polykov loop is not strictly zero in the low temperature region as expected in quenched QCD. This is certainly a consequence of our vertex ansatz which does not strictly represent quenched QCD but includes a (small) amount of unquenching effects.

\section{Concluding remarks}

In this letter we have determined the dual condensate or dressed Polyakov loop by solving the Dyson-Schwinger equations for the quark propagator on a compact manifold. This order parameter for center symmetry measures contributions from closed loops winding once around the time direction. We observe a significant rise of the dressed Polyakov loop around and above the deconfinement transition temperature together with a significant decrease of the ordinary quark condensate. The angular dependence of the quark condensate shows a characteristic dependence of the quark mass: the lighter the quark the more contributions arise from loops with winding numbers larger than one. An obvious next step is to investigate what happens to the transition temperatures when the backreaction of the quarks onto the Yang-Mills sector is included. Particularly interesting in this respect is the case $N_{f}=2+1$ which recently is a subject of intense debate [20, 21] in the lattice community.

\section{Acknowledgments}

I thank Falk Bruckmann, Christoph Gattringer, Jens Müller and Jan Pawlowski for discussions. I am grateful to Axel Maas for discussions and for making the lattice data of Ref. [17] available. This work has been supported by the Helmholtz-University Young Investigator Grant number VH-NG-332.
[1] F. Karsch, arXiv:hep-lat/9903031.

[2] C. Gattringer, Phys. Rev. Lett. 97 (2006) 032003.

[3] F. Bruckmann, C. Gattringer and C. Hagen, Phys. Lett. B 647 (2007) 56.

[4] F. Synatschke, A. Wipf and C. Wozar, Phys. Rev. D 75 (2007) 114003;

[5] E. Bilgici, F. Bruckmann, C. Gattringer and C. Hagen, Phys. Rev. D 77 (2008) 094007.

[6] F. Synatschke, A. Wipf and K. Langfeld, Phys. Rev. D 77 (2008) 114018.

[7] J. Braun, H. Gies and J. M. Pawlowski, arXiv:0708.2413 [hep-th]; F. Marhauser and J. M. Pawlowski, arXiv:0812.1144 [hep-ph].

[8] A. Bender, D. Blaschke, Y. Kalinovsky and C. D. Roberts, Phys. Rev. Lett. 77 (1996) 3724.

[9] A. M. Polyakov, Phys. Lett. B 72 (1978) 477; L. Susskind, Phys. Rev. D 20, 2610 (1979).

[10] F. Bruckmann, C. Hagen, E. Bilgici and C. Gattringer, PoS LATTICE2008 (2008) 262.

[11] J. Danzer, C. Gattringer and A. Maas, JHEP 0901 (2009) 024.
[12] C. D. Roberts and S. M. Schmidt, Prog. Part. Nucl. Phys. 45, S1 (2000); A. Maas, J. Wambach and R. Alkofer, Eur. Phys. J. C 42 (2005) 93.

[13] J. Braun and H. Gies, JHEP 0606 (2006) 024.

[14] R. Alkofer, C. S. Fischer, F. J. Llanes-Estrada and K. Schwenzer, Annals Phys. 324 (2009) 106.

[15] C. S. Fischer, R. Alkofer and H. Reinhardt, Phys. Rev. D 65 (2002) 094008; C. S. Fischer and M. R. Pennington, Phys. Rev. D 73 (2006) 034029; C. S. Fischer, A. Maas, J. M. Pawlowski and L. von Smekal, Annals Phys. 322 (2007) 2916.

[16] C. S. Fischer, A. Maas and J. M. Pawlowski, arXiv:0810.1987 [hep-ph].

[17] A. Cucchieri, A. Maas and T. Mendes, Phys. Rev. D 75, 076003 (2007).

[18] C. S. Fischer and R. Williams, Phys. Rev. D 78 (2008) 074006.

[19] F. Bruckmann, C. Gattringer and C. Hagen, private communication.

[20] Y. Aoki et al., arXiv:0903.4155 [hep-lat].

[21] A. Bazavov et al., arXiv:0903.4379 [hep-lat]. 